\title{
Resonant electromagnetism in photonic crystals composed of triangular-arrayed rods with both dielectric constant and magnetic permeability functions
}

\author{
S. Y. Yang \\ Institute of Electro-optical Science and Technology, National Taiwan Normal University, 88, Section 4, Ting-Chou \\ Road, Taipei 116, Taiwan
}

Chin-Yih Hong and I. Drikis

Department of Mechanical And Automation Engineering, Da-Yeh University, 112, Shan-Jiau Road, Da-Tusen, ChangHwa 515, Taiwan

H. E. Horng

Department of Physics/Institute of Electro-optical Science and Technology, National Taiwan Normal University, 88, Section 4, Ting-Chou Road, Taipei 116, Taiwan

H. C. Yang

Department of Physics, National Taiwan University, 1, Section 4, Roosevelt Road, Taipei 106, Taiwan

Received March 14, 2003; revised manuscript received September 1, 2003; accepted September 16, 2003

The modified frequency-domain method was used to simulate the photonic properties of triangular-arrayed rods surrounded by air and possessing nonunity dielectric and magnetic permeability functions. It was found that the photonic bandgap becomes broader for the TE mode when material with higher magnetic permeability and lower dielectric constant is used for rods as compared with that of purely dielectric rods with the same refractive index, whereas the gap is reduced for the TM mode. We further examined the photonic characteristics of the photonic-crystal cavity formed when a point defect is introduced into the rod array. With a fixed refractive index of the rods, the resonant frequency of the TE mode in the first band gap is lower and the electromagnetic energy concentration at the point defect is higher when material with higher magnetic permeability and lower dielectric constant is used for the rods. The opposite was found for the TM mode. (C) 2004 Optical Society of America

OCIS codes: 230.4320, 000.4430, 230.2090, 230.3810.

A photonic crystal is a system of periodic variation in the refractive-index contrast in a space. The period of a photonic crystal ranges from submicrometer to micrometer. As a result of the periodic variation in refractive index contrast, and assuming an adequate period, bandlike dispersion relations-the so-called photonic band structures-result for electromagnetic waves propagating in the photonic crystal. ${ }^{1,2}$ Furthermore, when defects are introduced into the photonic crystal, guided modes are generated in the forbidden portion of the photonic band structure of the original defect-free photonic crystal. Thus, light of certain frequencies can be localized about the defects. ${ }^{3-5} \quad$ By taking advantage of this particular property, photonic crystals have been further developed as gap-guided waveguides, ${ }^{3}$ microcavities, ${ }^{4}$ and so on. These devices have promising applications in electrooptics, such as in telecommunications, laser engineering, and integrated optical circuits.

Owing to well-established lithographical techniques for semiconductors, most photonic crystals are made of these materials. ${ }^{3-5}$ However, some researchers have used polymers to fabricate photonic crystals in view of their simpler fabrication and lower cost. ${ }^{6,7}$ Regardless how a photonic crystal is made, the periodic refractive-index contrast is attributed to the periodically distributed dielectric constant function, while the magnetic permeability is unity throughout the crystal. The influence of the contrast in dielectric constant and other properties of photonic band structures can be analyzed numerically by solving the master equation of magnetic field intensity $\mathbf{H}$ for the dielectric materials. ${ }^{8}$

Recently, magnetic materials have become increasingly important in electro-optics because of their tunable magneto-optical properties and other interesting phenomena that are absent in dielectric photonic crystals. ${ }^{9-11}$ For example, Saado et al. have experimentally demonstrated photonic crystal composed of self-assembled macroclusters of floating magnetic particles that are tunable simply by varying the external magnetic field strength. ${ }^{9}$ Figotin and Vitebsky have indicated that one can con- 
struct a magnetic photonic crystal with a strongly asymmetric dispersion relation. ${ }^{10}$ This asymmetry results in one-way transparency for the crystal. According to another report, ${ }^{11}$ the size of the bandgap can be reduced to achieve single-mode waveguides or cavities for utilizing magnetic material in photonic crystals. We believe that more new and interesting phenomena could be discovered by studying magnetic photonic crystals.

In previous studies by some of us and others, ${ }^{12,13}$ a tunable, triangular-ordered structure that may be used in photonic crystals was achieved in a magnetic fluid film under external magnetic fields. Although the effect of the magnetic permeability on the photonic properties has been discussed for the diamond-ordered structure ${ }^{14}$ and for a one-dimensional periodic structure, ${ }^{15}$ its effects on the triangular structure obtained in the magnetic fluid films is still unclear. In the present work, to clarify the role of magnetic permeability in photonic properties, we investigated numerically the photonic characteristics of triangular-arrayed magnetic rods in air by the modified frequency-domain simulation method, which calculates the eigenmodes propagating in the magnetic photonic crystals. $^{16}$

The modified frequency-domain method ${ }^{16}$ was used to simulate the photonic properties of triangular-arrayed rods in air. Since both the dielectric constant $\varepsilon_{\text {rod }}$ and magnetic permeability $\mu_{\text {rod }}$ functions of rods may vary periodically with position in this case, the following master equation for the magnetic field $\mathbf{B}$ in the photonic crystal is considered.

$$
\left(\frac{1}{\mu} \nabla\right)\left(\frac{1}{\epsilon} \nabla\right)\left(\frac{1}{\mu} \mathbf{B}\right)=\left(\frac{\omega}{c}\right)^{2}\left(\frac{1}{\mu} \mathbf{B}\right) .
$$

It is important to note that both sides of master Eq. (1) have transverse as well as longitudinal wave components. However, because the longitudinal modes are not independent but can be expressed in terms of the transverse ones, only the transverse components in Eq. (1) need be considered. Consequently, the master equation of the system becomes

$$
\mathbf{P}_{\perp}\left(\frac{1}{\mu} \nabla\right)\left(\frac{1}{\epsilon} \nabla\right)\left(\frac{1}{\mu} \mathbf{B}\right)=\left(\frac{\omega}{c}\right)^{2} \mathbf{P}_{\perp}\left(\frac{1}{\mu} \mathbf{B}\right),
$$

where $\mathbf{P}$ denotes the projection operator to transverse basis, such that

$$
\mathbf{P}_{\perp} \mathbf{F}=\exp (i k r) \sum_{\mathbf{G}}\left(a_{\mathbf{G}}^{u} e_{\mathbf{G}}^{u}+a_{\mathbf{G}}^{\nu} e_{\mathbf{G}}^{\nu}\right) \exp (i \mathbf{G r} r) .
$$

we designate $f_{\mathbf{G}}^{u}$ and $f_{\mathbf{G}}^{\nu}$ as expansion coefficients to transverse wave basis of any field vector $\mathbf{F}$ with the general form

$$
\mathbf{F}=\exp (i k r) \sum_{\mathbf{G}}\left(f_{\mathbf{G}}^{u} e_{\mathbf{G}}^{u}+f_{\mathbf{G}}^{\nu} e_{\mathbf{G}}^{\nu}+f_{\mathbf{G}}^{\|} e_{\mathbf{G}}^{\|}\right) \exp (i \mathbf{G} r)
$$

where $e_{\mathbf{G}}^{u}, e_{\mathbf{G}}^{\nu}$, and $e_{\mathbf{G}}^{\|}$are orthogonal unit vectors with $e_{\mathbf{G}}^{u}$ and $e_{\mathbf{G}}^{\nu}$ being normal and $e_{\mathbf{G}}^{\|}$being parallel to the wave vector $\mathbf{k}+\mathbf{G}$. Remarkably, master Eq. (2) may be referred to as the generalized eigenproblem and can be solved by using the Bloch-Rayleigh-quotient method ${ }^{17}$ since the Bloch expansion is used in Eqs. (3) and (4) so that the solutions have the same periodicity. To accelerate the iteration process for finding the eigenvectors of Eq. (2), an adequate preconditioning operator is needed. ${ }^{8}$ Because the left-hand operator of Eq. (2)—denoted by $A$ here-cannot be inverted simply by reversing each of the steps in computing $A \mathbf{B}$, and also because the dielectric constant and magnetic permeability are periodically piecewise constant in space, we introduce the approximate $A$ (denoted as $\widetilde{A}$ ) to obtain

$$
\widetilde{A} \mathbf{B}=\mathbf{P}_{\perp}\left(\frac{1}{\mu} \nabla\right) \mathbf{P}_{\perp}\left(\frac{1}{\epsilon} \nabla\right) \mathbf{P}_{\perp}\left(\frac{1}{\mu} \mathbf{B}\right) .
$$

Details of the modified frequency-domain method are given in Ref. 16.

The photonic band structures of the triangular-arrayed rods in air for TE and TM modes are shown in Figs. 1 and 2 , respectively. The ratio of the rod radius to the rod spacing is set at 0.2. The solid curves in Figs. 1(a) and 1 (b) give the band structures of TE modes for the dielectric rods, for example, $\left(\epsilon_{\mathrm{rod}}, \mu_{\mathrm{rod}}\right)=(10,1)$. It is clear that there is no bandgap for TE modes in the present situation. The variation in the photonic band structure due to the enhancement in the refractive-index contrast between the rods and the air is investigated. Since the refractive index of the rods $n_{\text {rod }}$ can be raised by increasing either $\mu_{\text {rod }}$ or $\epsilon_{\text {rod }}$ by means of the equation $n_{\text {rod }}$ $=\left(\mu_{\text {rod }} \epsilon_{\text {rod }}\right)^{1 / 2}$, the effects on the photonic band structure attributed to the variables $\epsilon_{\text {rod }}$ and $\mu_{\text {rod }}$ are discussed separately. Hereafter, the case with increasing $\mu_{\text {rod }}$ with fixed $\epsilon_{\text {rod }}(=10)$ is denoted Case I, while Case II denotes increasing $\epsilon_{\text {rod }}$ with fixed $\mu_{\text {rod }}(=1)$.

The results of Case I for TE modes are shown in Fig. 1(a). When $\mu_{\text {rod }}$ is increased from 1 , a bandgap occurs for the TE modes, and the forbidden band is shifted toward the lower-frequency region. The $\mu_{\text {rod }}$ dependence of the first two bandgaps for TE modes in Case I are plotted with dashed curves in Fig. 1(c), where the region enveloped by the dashed curves corresponds to the forbidden band of Case I. The second photonic bandgap at higher frequencies $\approx 0.9$ is not shown in Fig. 1(a). It was found that the first bandgap width increases with increasing $\mu_{\text {rod }}$, then becomes unchanged when $\mu_{\text {rod }}$ is larger than 2.1, as shown by the dashed curve in the inset of Fig. 1(c).

For Case II, a similar variation in the photonic band structure of TE modes to that in Case I can also be obtained, as shown in Fig. 1(b). The corresponding $\epsilon_{\text {rod }}$ dependence of the first two bandgaps of TE modes is within the region surrounded by the solid curves in Fig. 1(c). The $\epsilon_{\text {rod }}$-dependent width of the first bandgap is plotted with the solid curve in the inset of Fig. 1(c). It shows a different behavior from the $\mu_{\text {rod }}$-dependent gap width. It is obvious that a broader photonic bandgap under a fixed $n_{\text {rod }}\left[=\left(\epsilon_{\text {rod }} \mu_{\text {rod }}\right)^{1 / 2}\right]$ is exhibited for Case I when compared with Case II.

As for the TM modes, the photonic band structures for Case I with a fixed $\epsilon_{\text {rod }}$ of 10 and Case II with a fixed $\mu_{\text {rod }}$ of 1 are given in Figs. 2(a) and 2(b), respectively. The $\epsilon_{\text {rod }}-\mu_{\text {rod }}$ dependence of the first two bandgaps for the TM modes is shown in Fig. 2(c) for both cases. It was found that no matter whether $\epsilon_{\text {rod }}$ or $\mu_{\text {rod }}$ is increased to enhance the refractive-index contrast between the rod and 

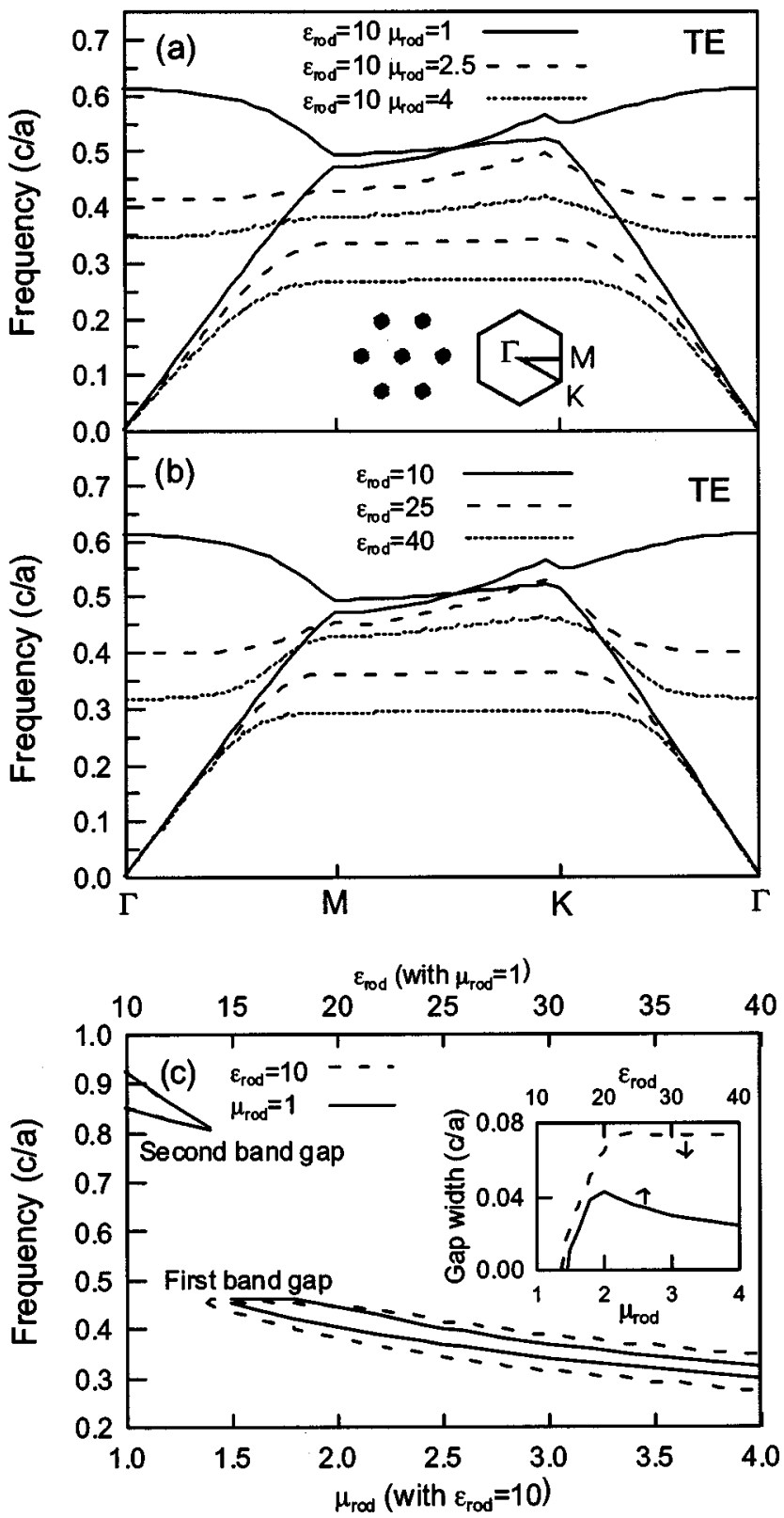

Fig. 1. Photonic band structures for TE modes propagating in the triangular-arrayed rods with various (a) magnetic and (b) dielectric permeabilities in air. The inset in (a) presents schematically the triangular-arrayed rods in real and in reciprocal space. The ratio of rod radius to rod spacing is set at 0.2 . The magnetic permeability $\mu_{\text {rod }}$ in (b) is unity in all cases. The $c$ and $a$ in the vertical axis labels of panels (a) and (b) denote the light speed and the rod spacing, respectively. The $\mu_{\text {rod }}{ }^{-}$and $\epsilon_{\text {rod }}$-dependent photonic bandgaps of the TE modes are shown in panel (c). The photonic bandgaps in the regions enveloped by the dashed curves in (c) correspond to Case I, in which the $\mu_{\text {rod }}$ increases and the $\epsilon_{\text {rod }}$ is kept at 10. The band gaps enveloped by the solid curves are for Case II with a fixed $\mu_{\text {rod }}(=1)$ and $\epsilon_{\text {rod }}$ varying from 10 to 40 .

the air, the forbidden band moves to the region with lower frequencies. Also, the first bandgaps in both cases become somewhat narrow, as shown in the inset of Fig. 2(c), while the second and third bandgaps for Case II become broader. Moreover, the photonic bandgap for Case I is narrower than the corresponding bandgap for Case II pos- sessing the same contrast in refractive index between the rod and air. This result is opposite from that for the TE modes shown in Fig. 1(c).

After realizing the photonic band structures of the triangular-arrayed rods in air for various rod refractive indices by varying either the dielectric constant or the magnetic permeability, we investigate the resonant mode of the photonic-crystal cavity when a point defect is introduced into the triangular rod array. A top view of the geometric scheme of the photonic-crystal cavity is shown as the inset of Fig. 3(b). In the following, we use various values of $\epsilon_{\text {rod }}$ and $\mu_{\text {rod }}$ under the constraint of $\epsilon_{\text {rod }} \mu_{\text {rod }}$
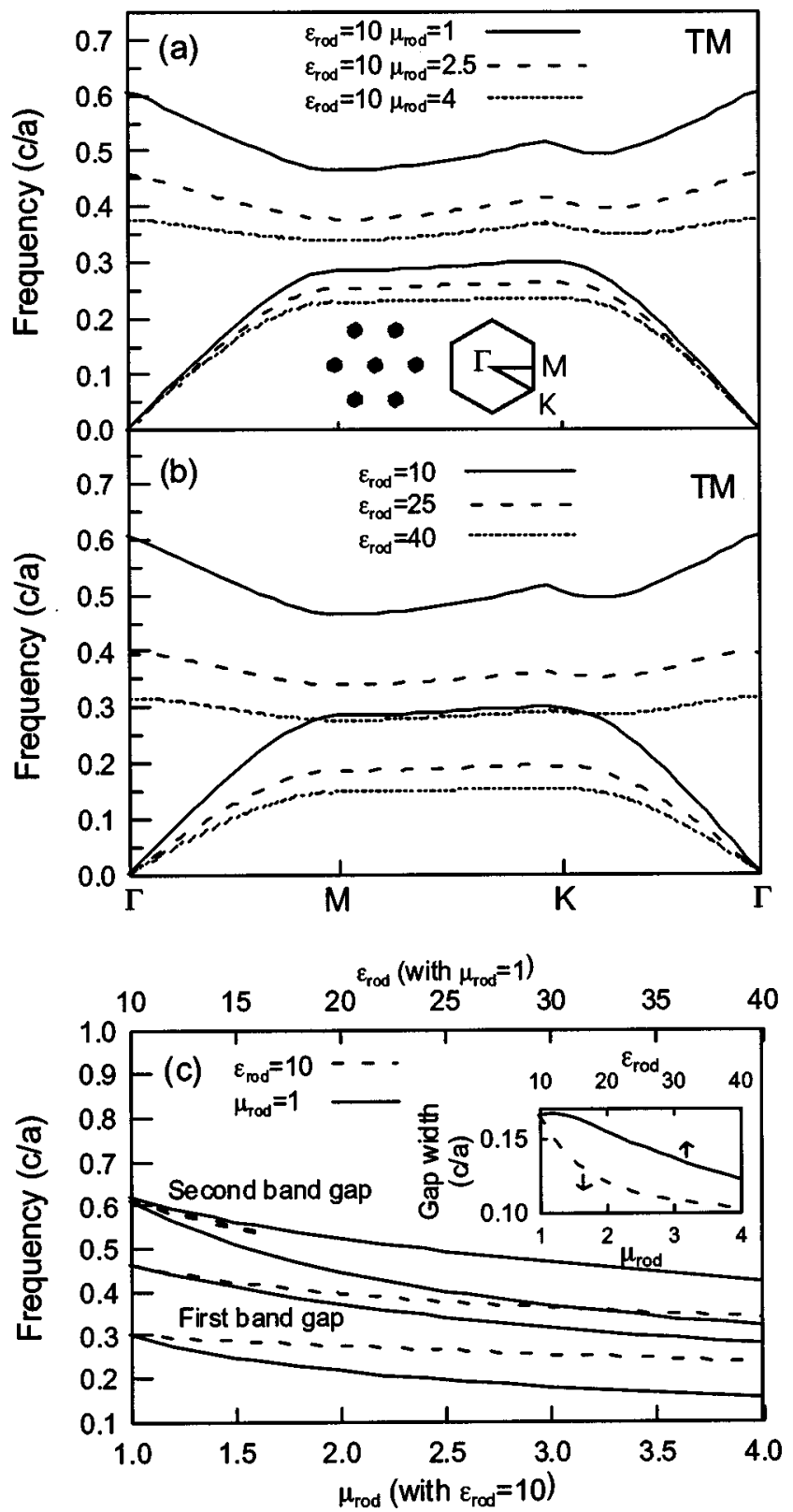

Fig. 2. Photonic band structures for TM modes propagating in the triangular-arrayed rods with various (a) magnetic and (b) dielectric permeabilities in air. The ratio of rod radius to rod spacing is set at 0.2 . The magnetic permeability $\mu_{\text {rod }}$ in (b) is unity in all cases. The $\mu_{\mathrm{rod}^{-}}$and $\epsilon_{\mathrm{rod}}$-dependent photonic bandgaps of the TM modes are shown in panel (c). The photonic bandgaps in the regions enveloped by the dashed (solid) curves correspond to Case I (Case II). 

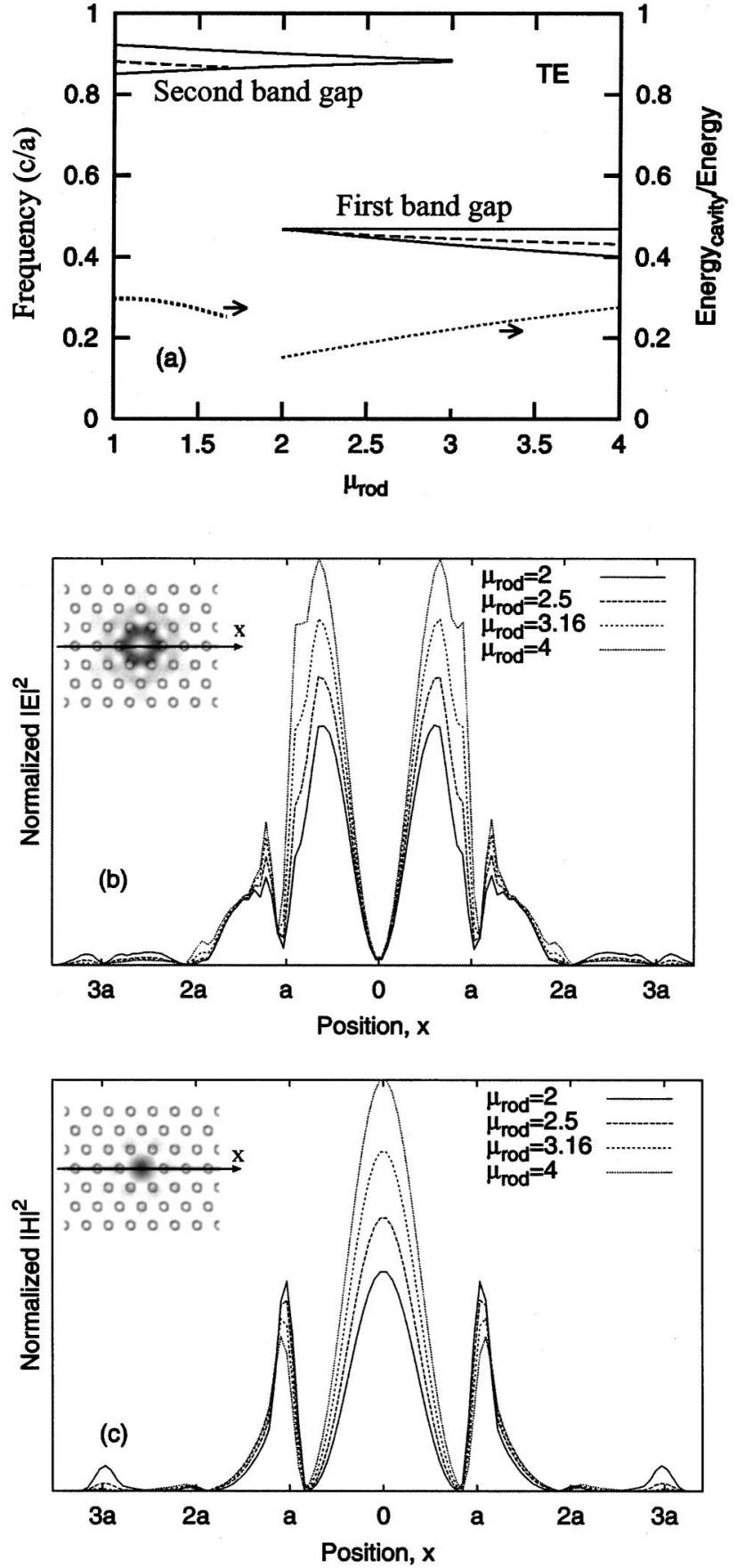

Fig. 3. (a) Photonic bandgaps (enveloped by solid curves) and resonant frequency (denoted by the dashed curves in gaps) versus magnetic permeability of rods for TE modes propagating in the triangular-arrayed rods in air with a point defect. The refractive index of the rods is fixed at 10 with varying $\mu_{\text {rod }}$. Distributions of the square of the amplitude of the electric field $|\mathbf{E}|^{2}$ and the magnetic field $|\mathbf{H}|^{2}$ for the resonant TE modes in the first bandgap around the point defect for various values of $\mu_{\text {rod }}$ are shown in panels (b) and (c), respectively. The insets in (b) and (c) show the geometry of the photonic-crystal cavity with a point defect at center. The distributions in (b) and (c) are analyzed along the lines in the insets labeled $x$. The fraction Energy $_{\text {cavity }} /$ Energy of the electromagnetic energy in the region of the defect is plotted as a function of $\mu_{\text {rod }}$ in panel (a).
$=10$ to calculate the resonant mode and its electromagnetic energy distribution in the cavity, and then determine the preferred materials to be used for the rods with a fixed contrast in refractive index with respect to the surrounding air.

In Fig. 3(a), the regions enveloped by solid curves denote the first and the second photonic bandgaps of the originally perfect triangular-arrayed rods in air. The frequencies of the resonant TE modes as functions of the rod magnetic permeability $\mu_{\text {rod }}$ are displayed by the dashed curves in the gaps. Note that the dielectric constant $\epsilon_{\text {rod }}$ is simultaneously reduced as the magnetic permeability $\mu_{\text {rod }}$ is increased to keep $n_{\text {rod }}^{2}$ constant (=10 here). The resonant frequency in the first bandgap is reduced from 0.4659 to 0.428 as $\mu_{\text {rod }}$ increases from 2 to 4 . As shown in this figure, the first bandgap does not exist for $\mu_{\text {rod }}$ $<2$. The distributions at the cavity for the square of the amplitude $|\mathbf{E}|^{2}$ of the electric field of the resonant TE modes in the first bandgap are shown in Fig. 3(b) for various values of $\mu_{\mathrm{rod}}$. The distribution is analyzed along the guided line shown in the inset of Fig. 3(b) with the origin of the $x$ axis in Fig. 3(b) located at the point defect of the triangular-arrayed rods. All the curves in Fig. 3(b) exhibit a similar behavior for various values of $\mu_{\text {rod }}$ : There exists a minimum at the point defect and most of the electric field is concentrated beside the rods around the point defect. However, $|\mathbf{E}|^{2}$ of the resonant TE modes at the region enveloped by the six rods nearest the point defect goes up significantly for a higher $\mu_{\text {rod }}$. This is also true for the square of the amplitude $|\mathbf{H}|^{2}$ of the magnetic field for the resonant TE modes, as shown in Fig. 3(c). But each $|\mathbf{H}|^{2}$ curve with respect to $x$ in Fig. 3(c) exhibits the maximum at the point defect instead of the minimum as for $|\mathbf{E}|^{2}$. The fraction Energy cavity $_{\text {chergy of the elec- }}$ tromagnetic energy in this region is further calculated for various $\mu_{\text {rod }}$, and the result is plotted with the dotted curves in Fig. 3(a). The Energy cavity $_{\text {/Energy of the reso- }}$ nant TE mode in the first band gap shows a nearly linearly increasing trend as $\mu_{\text {rod }}$ increases. This result implies that the resonant TE electromagnetic energy concentration around the point defect in the triangulararrayed rods in air can be enhanced by materials with higher magnetic permeability and lower dielectric constant for rods under the condition of fixed contrast in the refractive index between rods and surrounding air. Therefore, we can say that magnetic materials are preferred for the rods.

On the other hand, the resonant TM modes exhibit different properties from those of TE modes. It is shown in Fig. 4(a) that the resonant frequency of the TM modes in the first forbidden gap increases from 0.4048 to 0.4539 as $\mu_{\text {rod }}$ increases from unity to 4 and $\epsilon_{\text {rod }}$ decreases from 10 to 2.5. Furthermore, most of the electric field of the resonant TM modes is focused at the point defect. This is evidenced by the amplitude distribution $|\mathbf{E}|^{2}$ of the electric field in the photonic-crystal cavity shown in Fig. 4(b). On the other hand, the spatial distribution of $|\mathbf{H}|^{2}$ concentrates around the rim formed by the rods surrounding the point defect, as shown in Fig. 4(c). Both $|\mathbf{E}|^{2}$ and $|\mathbf{H}|^{2}$ within the defect region are reduced with increasing $\mu_{\text {rod }}$. This leads to a reduction in the energy fraction Energy $_{\text {cavity }} /$ Energy for a higher $\mu_{\text {rod }}$. The dotted curve 

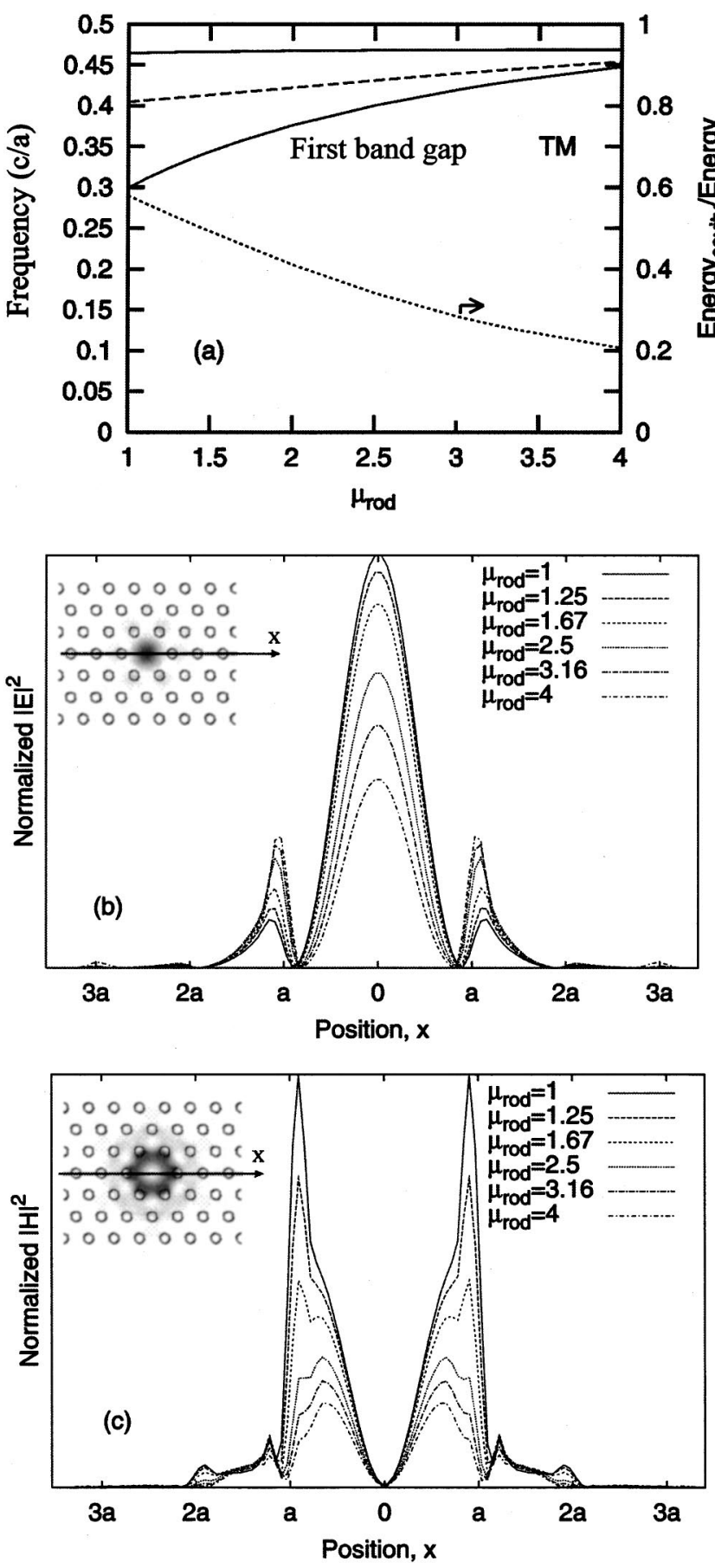

Fig. 4. (a) Photonic bandgaps (enveloped by solid curves) and resonant frequency versus magnetic permeability of rods for TM modes propagating in the triangular-arrayed rods in air with a point defect. The refractive index of the rods is fixed at 10 with varying $\epsilon_{\text {rod }}$. Distributions of the square of the amplitude of the electric field $|\mathbf{E}|^{2}$ and the magnetic field $|\mathbf{H}|^{2}$ for the resonant TM modes in the first bandgap around the point defect for various values of $\mu_{\text {rod }}$ are shown in panels (b) and (c), respectively. The insets in (b) and (c) show the geometry of the photonic-crystal cavity with a point defect at the center. The distributions in (b) and (c) are analyzed along the lines in the insets labeled $x$. The fraction Energy $y_{\text {cavity }} /$ Energy of the electromagnetic energy in the region of the defect is plotted as a function of $\mu_{\text {rod }}$ in panel (a).

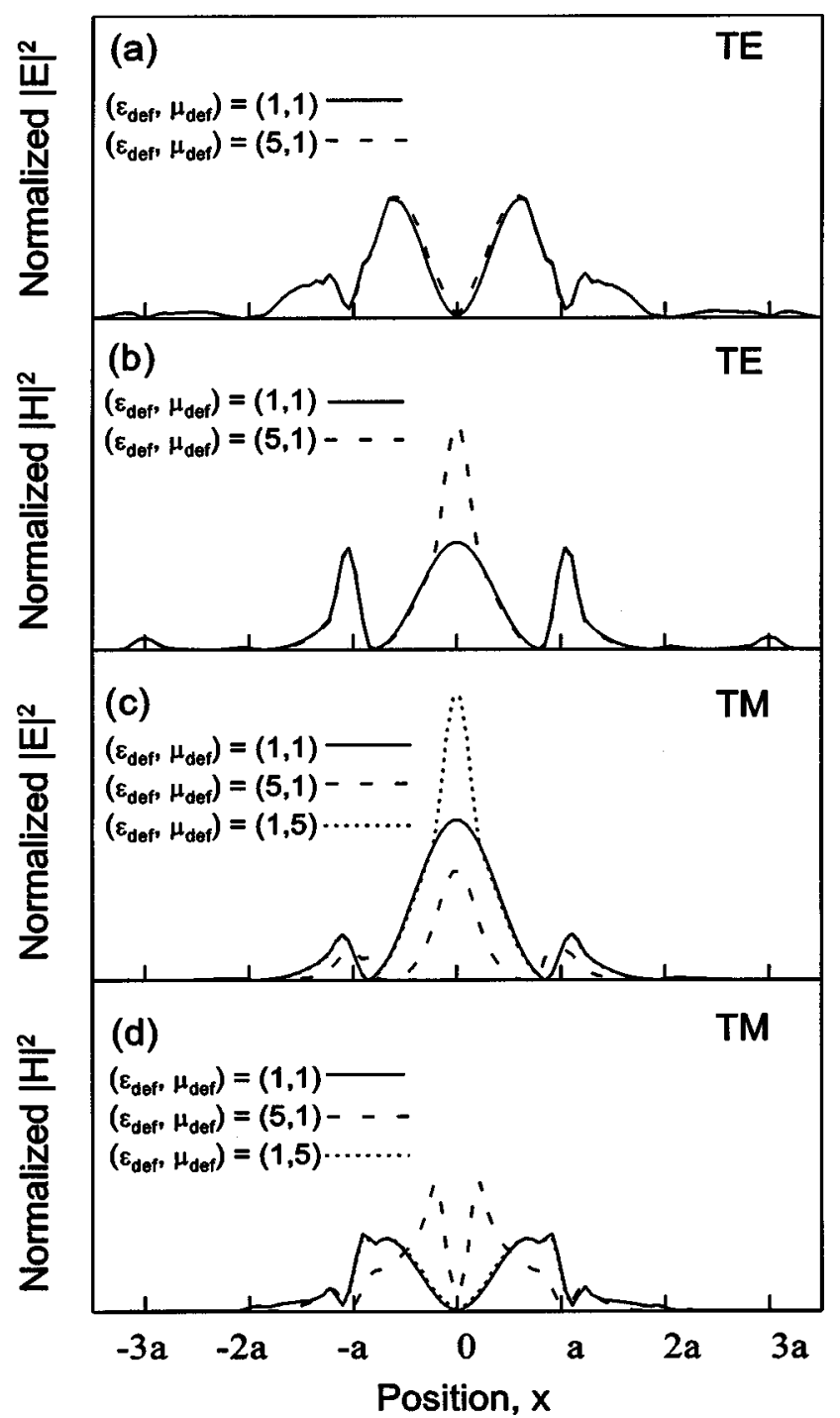

Fig. 5. Distributions of the square of the amplitude of the electric field $|\mathbf{E}|^{2}$ and the magnetic field $|\mathbf{H}|^{2}$ for the resonant [(a) and (b)] TE and [(c) and (d)] TM modes in the first bandgap around the point defect for various values of $\left(\epsilon_{\text {def }}, \mu_{\text {def }}\right)$. The $\left(\epsilon_{\text {rod }}, \mu_{\text {rod }}\right)$ is $(5,2)$ and the ratio of the rod-defect radius to the rod spacing is 0.2 .

in Fig. 4(a) quantitatively shows the reduction in Energy $_{\text {cavity }} /$ Energy with increasing $\mu_{\text {rod }}$ under a fixed $n_{\text {rod }}$. This implies that the dielectric rods are more suitable for resonating the TM modes in the cavity of triangular-arrayed rods in air.

We then investigated the electromagnetic properties of the resonant modes when a dielectric material is used for the defect of a photonic-crystal cavity instead of air. Without losing generality, we select $\left(\epsilon_{\text {rod }}, \mu_{\text {rod }}\right)$ as $(5,2)$ and the ratio of the defect radius to the rod spacing as 0.2 hereafter. Figures 5(a) and 5(b) show the $|\mathbf{E}|^{2}$ and $|\mathbf{H}|^{2}$ distributions of the resonant TE modes in the first bandgap. The solid curves correspond to the cavity with an air defect, while the dashed lines are for the dielectric defect with dielectric constant $\epsilon_{\text {def }}=5$ and magnetic permeability $\mu_{\mathrm{def}}=1$. It is obvious that the electromagnetic energy of the resonant TE mode is further concentrated at the defect when the air defect is replaced by a dielectric 


\section{Table 1. Frequency and Energy Concentration Energy $_{\text {cavity }} /$ Energy of the Resonant Modes in the First Bandgap ${ }^{a}$}

\begin{tabular}{|c|c|c|c|c|c|c|}
\hline \multicolumn{2}{|c|}{ Rod } & \multicolumn{2}{|c|}{ Defect } & \multirow[b]{2}{*}{ Polarization } & \multirow{2}{*}{$\begin{array}{l}\text { Resonant } \\
\text { Frequency }\end{array}$} & \multirow{2}{*}{$\begin{array}{c}\text { Energy }_{\text {cavity }} / \\
\text { Energy }\end{array}$} \\
\hline$\epsilon_{\text {rod }}$ & $\mu_{\text {rod }}$ & $\epsilon_{\text {def }}$ & $\mu_{\mathrm{def}}$ & & & \\
\hline \multirow[t]{2}{*}{5} & \multirow[t]{2}{*}{2} & \multirow[t]{2}{*}{1} & \multirow[t]{2}{*}{1} & $\mathrm{TE}$ & 0.4629 & 0.151 \\
\hline & & & & TM & 0.4224 & 0.411 \\
\hline \multirow[t]{2}{*}{5} & \multirow[t]{2}{*}{2} & \multirow[t]{2}{*}{5} & \multirow[t]{2}{*}{1} & $\mathrm{TE}$ & 0.4640 & 0.167 \\
\hline & & & & $\mathrm{TM}$ & 0.3702 & 0.265 \\
\hline \multirow[t]{2}{*}{5} & \multirow[t]{2}{*}{2} & \multirow[t]{2}{*}{1} & \multirow[t]{2}{*}{5} & $\mathrm{TE}$ & \multicolumn{2}{|c|}{ No resonant mode } \\
\hline & & & & $\mathrm{TM}$ & 0.4195 & 0.424 \\
\hline
\end{tabular}

${ }^{a}$ Values are for a photonic-crystal cavity composed of triangular arrayed rods and a point defect of air, dielectric or magnetic material; ratio of rod-defect radius to rod spacing is set at 0.2 .

defect. The calculated Energy cavity $_{\text {Energy and fre- }}$ quency of the resonant modes are listed in Table 1.

On the other hand, for TM modes the $|\mathbf{E}|^{2}$ and $|\mathbf{H}|^{2}$ distributions around the defect are depressed when the defect is made of dielectric material, as shown in Figs. 5(c) and $5(\mathrm{~d})$. However, the electromagnetic density of the resonant TM mode is significantly enhanced when a magnetic defect is introduced into the cavity. The relevant resonant frequency and Energy cavity $_{\text {Energy values are }}$ also listed in Table 1 for the TM modes. Therefore, according to the results in Figs. 4 and 5, the photonic crystal with triangular-arrayed dielectric-magnetic rods and a magnetic-dielectric point defect is preferred to resonate TM-TE modes.

In conclusion, the modified frequency-domain numerical simulation method can characterize photonic crystals composed of either dielectric or magnetic material. The individual influence of the dielectric constant and the magnetic permeability on the photonic properties of triangular-arrayed rods are analyzed and can be clearly distinguished. These results also provide important information for designing photonic crystals and resonant cavities with either magnetic or dielectric materials.

\section{ACKNOWLEDGMENTS}

This work is supported by the National Science Council of Taiwan under grants NSC 92-2212-E-212-011 and NSC 92-2112-M-003-010, and partially with 91-N-FA01-2-4-4.

Corresponding author C.-Y. Hong's e-mail address is cyhong@mail.dyu.edu.tw.

\section{REFERENCES}

1. J. D. Joannopoulos, R. D. Meade, and J. N. Winn, Photonic Crystals (Princeton University, Princeton, N.J., 1995).

2. M. Loncar, T. Doll, J. Vuckovic, and A. Schere, "Design and fabrication of silicon photonic-crystal optical waveguides," J. Lightwave Technol. 18, 1402-1411 (2000).

3. A. Mekis, J. C. Chen, I. Kurland, S. Fan, P. R. Villeneuve, and J. D. Joannopoulos, "High transmission sharp bends in photonic-crystal waveguides," Phys. Rev. Lett. 77, 37873790 (1996).

4. J. Sabarinathan, P. Bhattachayry, P.-C. Yu, S. Krishna, J. Cheng, and D. G. Steel, "An electrically injected, InAs/ GaAs, quantum-dot, photonic-crystal-microcavity, lightemitting diode," Appl. Phys. Lett. 81, 3876-3878 (2002).

5. J. C. Knight, J. Broeng, T. A. Briks, and P. St. J. Russell, "Photonic bandgap guidance in optical fibers," Science 282, 1476 (1998).

6. S. G. Romanov, T. Maka, C. M. Sotomayor Torres, M. Müller, and R. Zentel, "Photonic bandgap effects upon the light emission from a dye-polymer-opal composite," Appl. Phys. Lett. 75, 1057-1059 (1999).

7. C. Liguda, G. Böttger, A. Kuligk, R. Blum, M. Eich, H. Roth J. Kunert, W. Morgenroth, H. Elsner, and H. G. Meyer, "Polymer photonic-crystal slab waveguides," Appl. Phys. Lett. 78, 2434-2436 (2001).

8. S. G. Johnson and J. D. Joannopoulos, "Block-iterative frequency-domain methods for Maxwell's equations in a planewave basis," Opt. Express 8, 173-190 (2001).

9. Y. Saado, M. Golosovsky, D. Davidov, and A. Frenkel, "Tunable photonic bandgap in self-assembled clusters of floating magnetic particles," Phys. Rev. B 66, 195108 (2002).

10. A. Figotin and I. Vitebskiy, "Electromagnetic unidirectionality in magnetic photonic crystals," Phys. Rev. B 67, 165210 (2003).

11. C.-Y. Hong, I. Drikis, S. Y. Yang, H. E. Horng, and H. C. Yang, "Slab-thickness-dependent bandgap size of twodimensional photonic crystals with triangular-arrayed dielectric or magnetic rods," J. Appl. Phys. 94, 2188-2191 (2003)

12. S. Y. Yang, H. C. Yang, H. E. Horng, B. J. Huang, C.-Y. Hong, W. S. Tse, C. H. Hsieh, and C. N. Chang, "Tunable ordered structures for the potential photonic-crystal waveguides by using magnetic fluid films," in Proceedings of 7th Opto Electronics and Communications Conference (2002)

13. S. Y. Yang, H. E. Horng, C.-Y. Hong, H. C. Yang, M. C. Chou, C. T. Pan, and Y. H. Chao, "Control method for the tunable ordered structures in magnetic fluid microstrips,” J. Appl. Phys. 93, 3457-3460 (2003).

14. M. M. Sigalas, C. M. Soukoulis, R. Biswas, and K. M. Ho "Effect of the magnetic permeability on photonic band gaps," Phys. Rev. B 56, 959-962 (1997).

15. M. Inoue, K. Arai, T. Fujii, and M. Abe, "Magneto-optical properties of one-dimensional photonic crystals composed of magnetic and dielectric layers," J. Appl. Phys. 83, 67686770 (1998).

16. I. Drikis, S. Y. Yang, H. E. Horng, C.-Y. Hong, and H. C. Yang, "Modified frequency-domain method for simulating the electromagnetics in periodic magnetoactive systems," J. Appl. Phys. (to be published).

17. P. E. Gill, W. Murray, and M. H. Wright, Practical Optimization (Academic, London, 1981). 